\title{
Direct imaging and spectroscopy of planets around young stars: GQ Lupi
}

\author{
Ralph Neuhäuser ${ }^{1}$, Christopher Broeg ${ }^{1}$, Markus Mugrauer ${ }^{1}$ \\ and Eike Guenther ${ }^{2}$ \\ ${ }^{1}$ Astrophysikalisches Institut und Universitäts-Sternwarte, Schillergäßchen 2, D-07745 Jena, \\ Germany \\ emails: rne, broeg, markus@astro.uni-jena.de \\ ${ }^{2}$ Thüringer Landessternwarte Tautenburg, Sternwarte 5, D-07778 Tautenburg, Germany \\ email: guenther@tls-tautenburg.de
}

\begin{abstract}
Neuhäuser et al. (2005a) presented evidence for a sub-stellar, common proper motion companion to GQ Lup, which is almost certainly a planet imaged directly, based on two independent mass estimates by means of theoretical models. The magnitude of GQ Lup b was determined by relative photometry compared to GQ Lup A. During a one-month monitoring of the primary star, we found is to be a variable with $\mathrm{K}=6.9$ to $7.3 \mathrm{mag}$ (absolute photometry). Then, we re-observed GQ Lup A+b in May 2005, again with VLT/NACO. Due to the monitoring in April 2005, we could predict the exact K-band magnitude range of the primary during the new AO observation. Hence, we could determine a more precise error of the K-band magnitude of GQ Lup b by relative photometry compared to the primary. The new value is consistent with the previous estimate within the error margin, so that also the conclusions in Neuhäuser et al. $(2005 \mathrm{a}, \mathrm{b})$ about its very low mass remain valid.
\end{abstract}

Keywords. GQ Lup b, direct imaging

\section{Introduction}

Neuhäuser et al. (2005a) presented direct evidence for a companion of the $\leqslant 2 \mathrm{Myr}$ young classical T Tauri star GQ Lup in the Lupus star forming region at $140 \pm 50 \mathrm{pc}$ from imaging, astrometry, and spectroscopy. With direct K-band imaging using VLT/NACO, we detected an object 6 mag fainter than GQ Lup located $0.7^{\prime \prime}$ west of it. Compared to images obtained 2 to 5 years earlier with Subaru/CIAO and HST/PC, this object shares the proper motion of GQ Lup by 5 and $7 \sigma$, respectively, hence it is a co-moving companion. Its $K-L^{\prime}$ color is consistent with a spectral type early to mid L. Our NACO K-band spectrum yields spectral type M9-L4 with $\mathrm{H}_{2} \mathrm{O}$ and $\mathrm{CO}$ absorption, consistent with the new GAIA-Dusty template spectrum for $\log g \simeq 2$ to 3 and $\mathrm{T}_{\text {eff }} \simeq 2000 \mathrm{~K}$ with $\sim 1$ to $2 \mathrm{R}_{\text {jup }}$ radius at $\sim 140 \mathrm{pc}$, hence few Jupiter masses.

Using the core accretion formation model from Wuchterl et al. (2000) for planets, with some new estimates added, the mass is again around 1 to 3 Jupiter masses. Following the formation model for stars and brown dwarfs by direct gravitational collapse from Wuchterl \& Tscharnuter (2003), the mass is below 13 Jupiter masses. See fig. 4 in Neuhäuser et al. (2005a).

A comparison of our NACO K-band spectrum with the GAIA-dusty model (Brott \& Hauschildt, in preparation), an update of the AMES-dusty model, yielded the radius to be $1.2 \pm 0.5 R_{\text {Jup }}$ and the gravity to be $\log g=2.0$ to 3.3 (g in cgs units). For M9L4 spectral type, the temperature is $2050 \pm 450 \mathrm{~K}$. At the given flux of the GQLup companion $\left(\mathrm{K}_{\mathrm{s}}=13.10 \pm 0.15 \mathrm{mag}\right.$ at $140 \pm 50 \mathrm{pc}$ ), this yields a mass of $\leqslant 2 \mathrm{M}_{\mathrm{Jup}}$ (for $\log g \simeq 4$ and $2 \mathrm{R}_{\text {Jup }}$, it is $\sim 6 \mathrm{M}_{\text {Jup }}$ ). 
Mohanty et al. (2004a) measured gravities for isolated young brown dwarfs and freefloating planetary mass objects. Their coolest objects have spectral type M7.5 and gravities as low as $\log g=3.125$ (GG Tau Bb). This lead Mohanty et al. (2004b) to mass estimations as low as $\sim 10 \mathrm{M}_{\text {Jup }}$. GQ Lup A is younger than the Mohanty et al. Upper Sco objects. Its companion is at least as late in spectral type, probably even cooler. An object younger and cooler must be lower in mass. The GQ Lup companion is fainter than the faintest Mohanty et al. object (USco 128, $\sim 9 \mathrm{M}_{\mathrm{Jup}}$ ), so that the mass estimate for the GQLup companion would be $\leqslant 8 \mathrm{M}_{\text {Jup }}$ (Neuhäuser et al. 2005b). However, Reiners (2005) argue that some $\mathrm{TiO}$ oscillator strength were wrong in some inputs used in Mohanty et al. (2004a,b), so that all temperatures have to be increased by 150 to $200 \mathrm{~K}$, and, hence, also mass estimates. Therefore, the mass estimate for USco 128 and, hence, the upper mass limit for GQ Lup b is now $\sim 15 \mathrm{M}_{\mathrm{Jup}}$.

Mugrauer \& Neuhäuser (2005) then performed a more detailed astrometric analysis of the GQ Lup system, using all the (different) proper motions published for the primary. The common proper motion is significant in all cases, also when taking into account the error in parallax or distance $(140 \pm 50 \mathrm{pc})$. When using the weighted mean, the significance for common proper motion of GQ Lup and its companion is $6 \sigma+4 \sigma$ for no change in separation plus $7 \sigma$ for no change in position angle. They also discuss the question, whether GQLup and its common-proper motion companion are not bound, but share the same or similar proper motion as two independent members of the Lupus $\mathrm{T}$ association, which is a moving group, where most members should have the same motion anyway. This hypothesis can be rejected by several $\sigma$ : The probability to find by chance an L-dwarf fainter than $\mathrm{K}_{\mathrm{s}}=14 \mathrm{mag}$ within $0.7325^{\prime \prime}$ with (almost) the same proper motion of GQLup is only $\leqslant 3 \cdot 10^{-10}$. The orbital motion of the system is not yet detected $\left(1.4 \pm 2.2^{\prime \prime} \mathrm{mas} / \mathrm{yr}\right)$, but is probably smaller than the escape velocity $(5.3 \pm 2.1 \mathrm{mas} / \mathrm{yr})$, so that the system may well be gravitationally bound and stable. This is different for the companion of 2MASSWJ 1207334-393254 (2M1207), which is not yet shown to be bound and, if formed together, will not be stable. Also, for AB Pic A and its common proper motion companion, it is not yet shown that the remaining motion between them is smaller than the expected ejection velocity. However, if bound, then it is long-term stable, because the total mass or bounding energy is sufficient given the high mass of the primary.

\section{The variability of GQ Lup A and consequences for GQ Lup b}

In Neuhäuser et al. (2005a), the magnitude of GQ Lup b was determined by relative photometry compared to GQ Lup A, a brightness difference of $6.00 \pm 0.15$ mag in K. They did not take into account the possible variability of star A, but used its 2MASS value $(\mathrm{K}=7.096 \pm 0.020 \mathrm{mag})$. The variability of star A would introduce an additional uncertainty (or rather a small shift when knowing the phase and correcting for this effect) not taken into account in Neuhäuser et al. (2005a).

Now, we have taken a new image of GQ Lup A with b on 27 May 2005 between 03:30h and 04:17h UT, again using NACO at the VLT/Yepun, as in Neuhäuser et al. (2005a). We have reduced the data as in 2004 .

From an extensive photometric monitoring campaign of GQ Lup A during April 2005 with ANDICAM at CTIO in the filters R, I, J, and K, as well as quasi-simultaneous radial velocity monitoring with HARPS at the ESO $3.6 \mathrm{~m}$ on La Silla, we could obtain the amplitude of its K-band variation, few tenth of mag, probably due to rotational modulation by a spotted surface, typical for classical T Tauri stars. 
Hence, we can predict the K-band magnitude of GQ Lup A of $\mathrm{K}=6.9$ to $7.3 \mathrm{mag}$ for May 2005, i.e. shortly after the monitoring in April 2005, so that no long-term variability is expected. The absolute value (or rather range) is obtained from data of two photometric nights during our April 2005 monitoring by using three stars from 2MASS, which appeared to constant with respect to eachother, so that they can be assumed to long-term constant.

Now, with a the magnitude difference between GQ Lup A and b on the image from 27 May 2005 being $6.10 \pm 0.15 \mathrm{mag}$, and with $\mathrm{K}=6.9$ to $7.3 \mathrm{mag}$ for star A, we can obtain the magnitude of GQ Lup b to be $K=13.20 \pm 0.25 \mathrm{mag}$ (on 27 May 2005). Then, with B.C.K $=3.3 \pm 0.1 \mathrm{mag}$ and $140 \pm 50 \mathrm{pc}$, its luminosity will be $\log \mathrm{L} / \mathrm{L}_{\odot}=-2.41 \pm 0.43$ (instead of $-2.37 \pm 0.41$ previously). Given the large error on the distance and, hence, luminosity, this does not change the mass estimate according to Figure 4 in Neuhäuser et al. (2005a), which is still 1 to 3 Jupiter masses.

The problem of variability of the primary (GQ Lup A) probably also affects the Lband magnitude (of GQ Lup b), however, the variability amplitude in L is expected to be smaller than in $\mathrm{K}$, if due to spots.

Liu (this conference) presented his own observations of GQ Lup A and b. He determined narrow-band colors for GQ Lup b within the J-band with HST/Nicmos and broad-band colors from the ground; he then compared the GQ Lup b colors in a color-color diagram with M5 to L0 dwarfs (with spectral types known from ground-based observations). The position of GQ Lup b in that color-color diagram was consistent with those dwarfs, so that Liu derived the temperature of GQ Lup b to be 2200 to 2650 K. However, because Liu did not obtain the same narrow-band colors for L1-4 dwarfs, this temperature estimate has to be considered as upper limit (consistent with $\mathrm{T}=1600$ to $2500 \mathrm{~K}$ from Neuhäuser et al. 2005a).

Liu also obtained some of his magnitudes for GQ Lup b by relative photometry compared to A, but did not take into account the variability of GQ Lup A, so that it is not surprising, that some magnitudes varry from observation to observation. Liu then compared GQ Lup b with Burrows et al. (1997) and Baraffe et al. (2002) models, where the GQ Lup b error ellipse crosses the D burning mass limit. He also compared its location with young brown dwarf companions, concluding that they are all quite similar in lumonisity and temperature. However, one also needs to take into account that GQ Lup $\mathrm{b}$ is much younger than all brown dwarf companion candidates used in that comparison, hence lower in mass.

We would like to note that the same problem of possible variability of the primary can have occured in the determination of the magnitude and, hence, luminosity and mass, of companions of other young stars such as, most recently, 2M1207 (Chauvin et al. 2005a), AB Pic (Chauvin et al. 2005b), and AB Dor (Close et al. 2005), the latter known to be one of the most variable stars on the sky.

\section{Following the orbital motion of GQ Lup b around A}

With the new AO observation of GQ Lup A+b in May 2005, we can also check again for common proper motion and possibly orbital motion. See figure 1 .

The separation between GQ Lup A and b is still constant within small error bars, so that common proper motion is highly significant.

Orbital motion is not yet detected. Orbital motion would be detectable as follows: A new data point would need to be significantly different from the full line (constant separation), but within the dotted lines (i.e. orbital motion detected); as long as a data point is consistent with the full line, orbital motion would not yet be detected, and one 


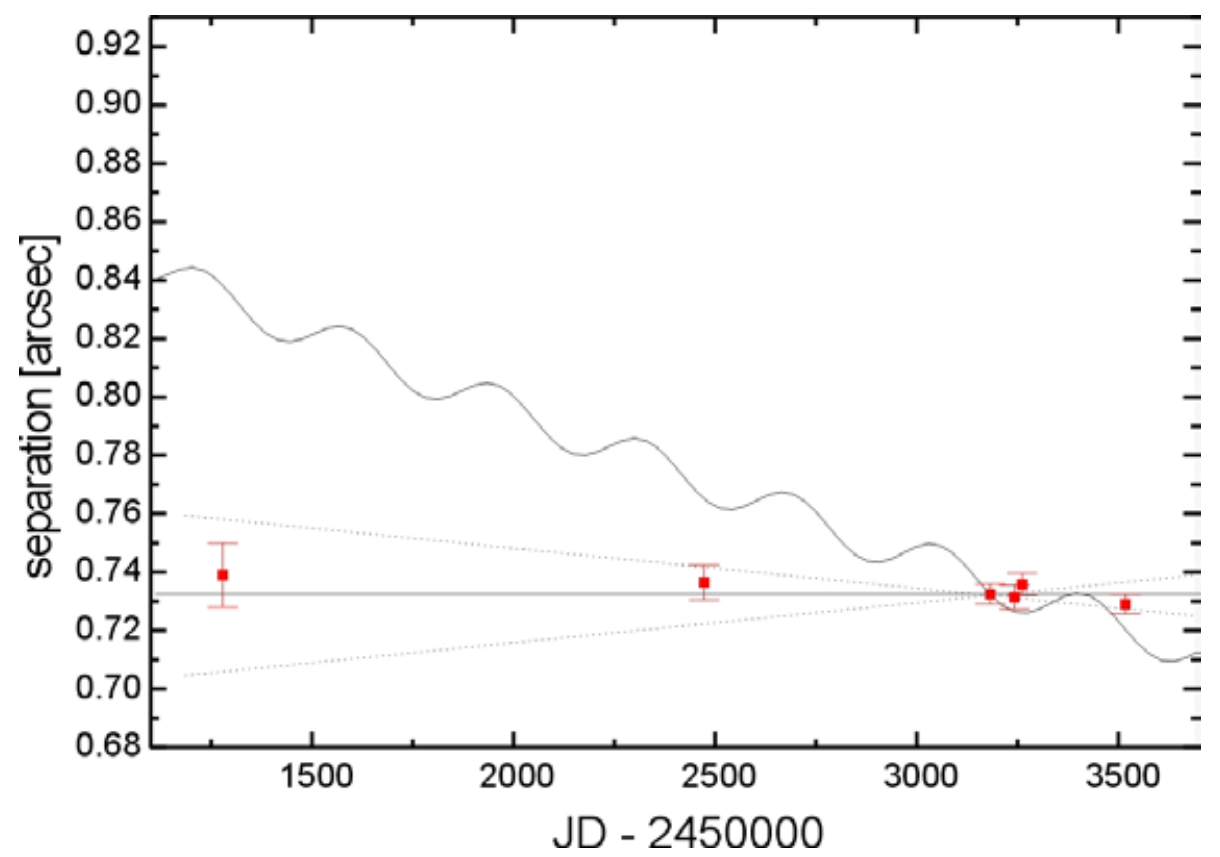

Figure 1. Observed separation between primary star GQ Lup A and its companion b with HST/PC (left), Subaru/CIAO (middle) and VLT/NACO (right, first the June 2004 image, then the Aug and Sept 2004 aquisition images, and finally our new data point from May 2005) compared to the expectation when bound (no change in separation as straight full line few mas/yr for orbital motion as straight dotted lines) or when background (motion of GQ Lup A alone to the SW due to its proper motion \pm its error with wobble due to expected parallactic motion of 7.1 mas). We can reject the background hypothesis by many $\sigma$.

would have to wait longer; if a data point would be on the curved upper lines, the fainter object would be a non-moving background object; if a data point would be anywhere else in the diagram, outside the dotted line cones, then it would not be bound.

The remaining possible differential motion between $\mathrm{A}$ and $\mathrm{b}$ is $1.4 \pm 2.2 \mathrm{mas} / \mathrm{yr}$. Based on the mass of GQ Lup A (0.7 solar masses) and GQ Lup b (negligible), we can expect orbital motion of $3.7 \pm 1.5 \mathrm{mas} / \mathrm{yr}$, which is hence detectable within a few years. In the other hand, if the system would not be bound, we would expect an ejection velocity of $\mathrm{b}$ on the order of $5.2 \pm 2.1 \mathrm{mas} / \mathrm{yr}$. The fact that the remaining motion is smaller than this expected escape velocity shows that GQ Lupi A+b is most certainly bound.

\section{Conclusions}

All the available evidence regarding GQ Lup b is consistent with it being a planet imaged directly (Neuhäuser et al. 2005a). Two other similar claims were published since then: 2M1207 (Chauvin et al. 2005a) and AB Pic (Chauvin et al. 2005b). In table 1, we compare all the available data for these three planets or planet candidates (adapted from Neuhäuser 2005, where the parameters were derived in a homogeneous way, but updated for GQ Lup b).

Mass determination of directly detected planets is obtained thru theoretical models. If the objects involved are young, then their formation is to be taken into account. This is not yet implemented in Tucson or Lyon models. 
Table 1. Parameters of three planet candidates

\begin{tabular}{|c|c|c|c|}
\hline \multirow[t]{2}{*}{ Parameter } & \multicolumn{3}{|c|}{ Objects } \\
\hline & GQ Lup b & 2M1207 b & AB Pic b \\
\hline distance $[\mathrm{pc}]$ & $\mid 140 \pm 50$ & $70 \pm 20$ & $47.3 \pm 1.8$ \\
\hline membership & Lupus I & TWA (?) & TucHorA \\
\hline age $[\mathrm{Myr}]$ & $\mid \leqslant 2$ & 5-12: & $30-40$ \\
\hline $\begin{array}{l}\text { epoch difference }[\mathrm{yr}] \\
\text { separation } \\
\text { significance for CPM (1) }[\sigma] \\
\text { remaining motion A/b [mas/yr] } \\
\text { orbital motion expected [mas/yr] } \\
\text { escape velocity expected [mas/yr] } \\
\text { long-term stable? (2) }\end{array}$ & \begin{tabular}{|l}
5 \\
$0.7 ", 100 \mathrm{AU}$ \\
$6+4+7$ \\
$1.4 \pm 2.2$ \\
$3.7 \pm 1.5$ \\
$5.2 \pm 2.1$ \\
yes
\end{tabular} & $\begin{array}{l}1 \\
0.8^{\prime \prime}, 54 \mathrm{AU} \\
2+2+4+4 \\
4.1 \pm 8.2 \\
1.9 \pm 0.6 \\
2.7 \pm 0.9 \\
\text { no }\end{array}$ & $\begin{array}{l}1.5 \\
0.5 ", 258 \mathrm{AU} \\
3+5 \\
6.9 \pm 13.2 \\
6.9 \pm 0.4 \\
9.8 \pm 0.6 \\
\text { yes }\end{array}$ \\
\hline $\begin{array}{l}\text { Spectral type } \\
\text { spectrum resolution } \\
\text { spectrum } \mathrm{S} / \mathrm{N} \text { ratio } \\
\mathrm{T}_{\text {eff }}[\mathrm{K}]\end{array}$ & \begin{tabular}{|l|} 
M9-L4 \\
700 \\
45 \\
$2520-1600$
\end{tabular} & \begin{tabular}{|l|} 
L5-L9.5 \\
$<700$ \\
low \\
$2000-1100$
\end{tabular} & \begin{tabular}{|l|} 
L0-3 \\
700 \\
high \\
$2400-1600$
\end{tabular} \\
\hline gravity $\log g[\mathrm{cgs}]$ & |2.0-3.3(3) & unknown & unknown \\
\hline radius $\left[\mathrm{R}_{\mathrm{jup}}\right]$ & $\mid 1.2 \pm 0.6(4)$ & unknown & unknown \\
\hline $\begin{array}{l}\mathrm{M}_{\mathrm{K}}[\mathrm{mag}] \\
\log L_{b o l} / \mathrm{L}_{\odot}\end{array}$ & $\begin{array}{l}7.47 \pm 0.94\left(^{*}\right) \\
-2.41 \pm 0.43\left(^{*}\right)\end{array}$ & $\begin{array}{l}12.70 \pm 0.75 \\
-4.49 \pm 0.34\end{array}$ & $\begin{array}{l}10.77 \pm 0.14 \\
-3.730 \pm 0.039\end{array}$ \\
\hline $\begin{array}{l}\text { mass }\left[\mathrm{M}_{\mathrm{jup}}\right] \text { Lyon/Tucson } \\
\text { mass }\left[\mathrm{M}_{\text {jup }}\right] \text { Wuchterl }\end{array}$ & $\mid \begin{array}{l}3-50 \\
1-3\end{array}$ & $\begin{array}{l}2-70 \\
\text { n/a (5) }\end{array}$ & $\begin{array}{l}11-70 \\
\mathrm{n} / \mathrm{a}(5)\end{array}$ \\
\hline
\end{tabular}

Remarks: $\left(^{*}\right)$ this paper; (1) significance for common proper motion in Gaussian $\sigma$; (2) according to criteria in Weinberg et al. (1987) and Close et al. (2003); (3) from fit to theoretical GAIAdusty template spectrum; (4) from fit to spectrum with flux and temperature known; (5) not applicable, because outside of plotted or calculated range.

Ref.: Neuhäuser et al. 2005a, Mugrauer \& Neuhäuser 2005, Chauvin et al. 2005a, Chauvin et al. 2005b, Neuhäuser et al. 2005c.

The model by Wuchterl \& Tscharnuter (2003) for stars and brown dwarfs does take into account their formation, so that it can be valid for very young objects. The tracks for planets shown in Fig. 4 in Neuhäuser et al. (2005a) are calculated based on the nucleated instability hypothesis (Wuchterl et al. 2000).

For higher confidence in the mass determination and, hence, classification as planets, one would need to test and possibly calibrate the theoretical models used, both the formation models (such as Wuchterl \& Tscharnuter 2003) as well as the atmospheric models (such as Peter Hauschildt's GAIA-dusty). Such tests can be done with objects, where the masses have been determined dynamically, e.g. transiting planets with direct or indirect (secondary eclipse) magnitude estimations, or resolved very low-mass binaries with solved orbits.

Alternatively, higher resolution higher $\mathrm{S} / \mathrm{N}$ spectra of the companions would also be helpfull to constrain gravity, temperature, radius, and, hence, their masses.

\section{Acknowledgements}

We would like to acknowledge many fruitful discussions with Guenther Wuchterl and Andreas Seifahrt. 


\section{References}

Chauvin G., Lagrange A.M., Dumas C., Zuckerman B., Mouillet D., Song I., Beuzit J.L., \& Lowrance P. 2005a, A\&A 438, L25

Chauvin G., Lagrange A.M., Zuckerman B., et al. 2005b, A\&SA 438, L29

Close L.M., Siegler N., Freed M., \& Biller B. 2003, ApJ 587, 407

Close L.M., Lenzen R., \& Guirado J.C. 2005, Nature 433, 286

Mohanty S., Basri G., Jayawardhana R., Allard F., Hauschildt P., \& Ardila D. 2004a, ApJ 609, 854

Mohanty S., Jayawardhana R., \& Basri G. 2004b, ApJ 609, 885

Mugrauer M. \& Neuhäuser R. 2005, AN 326, 701 (astro-ph/0509162)

Neuhäuser R., Guenther E.W., Wuchterl G., Mugrauer M., Bedalov A., \& Hauschildt P. 2005a, A\&A 435, L13

Neuhäuser R., Guenther E.W., \& Hauschildt P.H. 2005b, in: A. Richichi \& F. Paresce (ed.), ESO Workshop The power of optical/IR interferometry, in press (astro-ph/0506011)

Neuhäuser R. 2005, in: M. Petr-Goetzens (ed.), ESO Workshop Multiple Stars accross the H-R Diagram, in press

Reiners A. 2005, $A N$ in press (ULMSF proceedings, astro-ph/0510502)

Weinberg M.D., Shapiro S.L., \& Wasserman I. 1987, ApJ 312, 367

Wuchterl G. \& Tscharnuter W.M. 2003, A\& A 398, 1081

Wuchterl G. \& Guillot T., Lissauer J. 2000, in: V. Mannings et al. (eds.), in: Protostars and Planets IV, 1081

\section{Discussion}

Close: The magnitude of AB Dor $\mathrm{C}$ was obtained by relative photometry compared to $\mathrm{AB}$ Dor $\mathrm{A}$ and $\mathrm{B}$, whose magnitudes where known from 2MASS, so that I believe our magnitude of $\mathrm{AB}$ Dor $\mathrm{C}$ is correct, even though of the large and fast variability of $\mathrm{AB}$ Dor A.

NeuhäUsER: AB Dor B, as companion to the young and very variable AB Dor A, should also be young and variable. For relative photometry compared to young and variable stars, one needs to know the exact rotational period and magnitude amplitude, and the rotational phase of one's own and the 2MASS observations.

LIU: In previous comparisons of observed spectra of sub-stellar objects to AMES-dusty models, one has often underestimated the gravity. Why should this be different in your comparison of the GQ Lup b spectrum with the new GAIA-dusty model ?

NeuhäUsER: The GAIA-dusty model (Brott \& Hauschildt, in prep.) is an update of the AMES-dusty model (Allard et al. 2001) with improved molecular dissociation constants, more dust species with opacities, spherical symmetry, and a mixing length parameter $1.5 \cdot \mathrm{H}_{\mathrm{p}}$. In particular, the new model uses spherical symmetry, which is most important for young and sub-stellar objects as GQ Lup A and b with low gravities. Hence, we expect less problems with the gravities than in previous models. The gravity estimate from the GAIA-dusty model for GQ Lup b is consistent with both the estimate just from the CO-lines (using the Gorlova et al. (2003) calibration) and with the gravity obtained for GQ Lup b from the Wuchterl model using the observed luminosity and temperature (see figure 4 in Neuhäuser et al. 2005a). In September 2005, we have taken a higher resolution spectrum, $\mathrm{R}=4000$ in the K-band, with Sinfoni at the VLT, being reduced. 